\title{
qEEG Measures of Attentional and Memory Network Functions in Medical Students: Novel Targets for Pharmacopuncture to Improve Cognition and Academic Performance
}

\author{
Vasavi R. Gorantla', Vernon Bond Jr. ${ }^{2}$, James Dorsey ${ }^{3}$, Sarah Tedesco ${ }^{4}$, Tanisha Kaur ${ }^{5}$, \\ Matthew Simpson ${ }^{6}$, Sudhakar Pemminati ${ }^{7}$, Richard M. Millis ${ }^{1 *}$
}

${ }^{1}$ Department of Behavioural Sciences and Neuroscience, AUA College of Medicine, Antigua and Barbuda

2 Department of Recreation, Human Performance \& Leisure Studies and Exercise Science \& Human Nutrition Laboratory, Howard University Cancer Centre, Washington, DC 20060, United States of America

${ }^{3}$ Department of Recreation, Human Performance \& Leisure Studies and Exercise Science \& Human Nutrition Laboratory, Howard University Cancer Centre, Washington, DC 20060, United States of America

${ }^{4}$ AUA College of Medicine, Antigua and Barbuda

${ }^{5}$ AUA College of Medicine, Antigua and Barbuda

${ }^{6}$ AUA College of Medicine, Antigua and Barbuda

${ }^{7}$ Department of Medical Pharmacology, AUA College of Medicine, Antigua and Barbuda 8 Department of Medical Physiology,

AUA College of Medicine, Antigua and Barbuda

\section{Key Words}

Theta/beta ratio, frontal theta asymmetry, frontal beta asymmetry, academic performance, neural plasticity, quantitative electroencephalography (qEEG), pharmacopuncture

\begin{abstract}
Objectives: Attentional and memory functions are important aspects of neural plasticity that, theoretically, should be amenable to pharmacopuncture treatments. A previous study from our laboratory suggested that quantitative electroencephalographic (qEEG) measurements of theta/beta ratio (TBR), an index of attentional control, may be indicative of academic performance in a first-semester medical school course. The present study expands our prior report by extracting and analyzing data on frontal theta and beta asymmetries. We
\end{abstract}

Received: Jan 08, 2019 Reviewed: Jan 28, 2019 Accepted: Sep 03, 2019

(c) This is an Open-Access article distributed under the terms of the Creative Common Attribution Non-Commercial License (http://creativecommons.org/licenses/by-nc/4.0/) which permits unrestricted noncommercial use, distribution, and reproduction in any medium, provided the original work is properly cited.

( $)$ This paper meets the requirements of KS X ISO 9706, ISO 9706-1994 and ANSI/NISO Z39.48-1992 (Permanence of Paper) test the hypothesis that the amount of frontal theta and beta asymmetries (fTA, fBA), are correlated with TBR and academic performance, thereby providing novel targets for pharmacopuncture treatments to improve cognitive performance.

Methods: Ten healthy male volunteers were subjected to 5-10 min of qEEG measurements under eyes-closed conditions. The qEEG measurements were performed 3 days before each of first two block examinations in anatomy-physiology, separated by five weeks. Amplitudes of the theta and beta waveforms, expressed in $\mu \mathrm{V}$, were used to compute TBR, fTA and fBA. Significance of changes in theta and beta EEG wave amplitude was assessed by ANOVA with post-hoc t-testing. Correlations between TBR, fTA, fBA and the raw examination scores were evaluated by Pearson's product-moment coefficients and linear regression analysis.

Results: fTA and fBA were found to be negatively correlated with TBR $(\mathrm{P}<0.03, \mathrm{P}<0.05$, respectively $)$ and 
were positively correlated with the second examination score $(\mathrm{P}<0.03, \mathrm{P}=0.1$, respectively).

Conclusion: Smaller fTA and fBA were associated with lower academic performance in the second of two first-semester medical school anatomy-physiology block examination. Future studies should determine whether these qEEG metrics are useful for monitoring changes associated with the brain's cognitive adaptations to academic challenges, for predicting academic performance and for targeting phamacopuncture treatments to improve cognitive performance.

\section{Introduction}

Pharmacopuncture treatments using herbal ginger are reported to improve cognitive and memory deficits [1]. The brain's memory and attentional control networks are important components of neural plasticity, learning and academic performance [2]. Childhood academic performance data have been used to predict the predilection for cognitive impairment in adulthood [3]. Heretofore, research on the cognitive aspects of academic performance has been hindered by an inability to correlate measures of efficient learning with the functions of critical nodes and hubs within the brain's attentional control and working memory networks. This impediment to studying academic performance has been effectively circumvented by the advent of computer-based technologies for psychometric testing and for measuring electrical and metabolic functions of the cerebral cortex (e.g. quantitative EEG, functional MRI). Whereas functional MRI (fMRI) provides information about the metabolic activity of the brain, quantitative EEG (qEEG) is primarily a measure of electrical activity. Because of the tight coupling between metabolic and electrical signaling in the brain [4], each of the aforementioned technologies can be viewed as a surrogate for the other. An inexpensive qEEG recording may be useful as a surrogate for an expensive fMRI recording [5, 6]. qEEG appears to be a useful tool for diagnosing attention deficit disorder (ADD) syndromes [7]. Previous studies have shown that qEEG voltage amplitudes in the theta $(5-8 \mathrm{~Hz})$ and beta $(15-\mathrm{Hz})$ frequencies are indicative of working memory [8] and complex thinking [9] respectively, and that the theta/ beta ratio (TBR) is an indicator of attentional control [10]. High TBR is thought to be diagnostic of ADD [11].

Frontal interhemispheric theta asymmetry, wherein the theta wave amplitude is substantially greater in one hemisphere than the other, is reported to be indicative of declarative and episodic working memory [11, 12]. A previous report from our laboratory suggests that TBR may be negatively correlated with academic performance on the second of two first-semester medical school anatomy-physiology examinations [13]. The present study is, therefore, designed to expand our prior analysis, using the same subjects and database, to test the hypothesis that frontal theta and beta asymmetries are correlated with both TBR and academic performance. Such a finding would suggest a novel use of TBR, frontal theta and frontal beta asymmetries (fTA, fBA) as physiological biomarkers for pharmacopuncture treatments to improve cognitive deficits and academic performance.

\section{Material and methods 2.1. Subjects}

This study was approved by the American University of Antigua College of Medicine Research Committee, Antigua and informed consent was obtained from the study participants (February 2017). Of ten first-semester male medical students who were recruited for the study, nine completed the study.

\subsection{Overview of experimental procedures}

The students were subjected to 5-10 min of qEEG measurements at 19 standard electrode sites during eyes-closed (EC) conditions (Brain Master Discovery 20, Brain Master Technologies, Inc., Bedford, OH, USA). qEEG studies were done for each student volunteer 3 days before each of their first two medical school examinations covering first-semester anatomy and physiology (Block 1, Block 2, respectively). The block 1 and block 2 examinations were 5 weeks apart. qEEG measurements under EC conditions were performed with subjects seated upright in a darkened room at the same time of day (8-10 AM) and at the same place. Mean \pm standard deviations (SD) of the amplitudes, expressed in $\mu \mathrm{V}$, were measured for the following standard qEEG frequencies: delta $(2-4 \mathrm{~Hz})$, theta $(4-7 \mathrm{~Hz})$, alpha $(8-12 \mathrm{~Hz})$ and beta $(13-30 \mathrm{~Hz})$ after manual editing using the New Mind Maps online editing tool (New Mind Technologies, Roswell, GA, USA). TBR was computed as the quotient of mean theta/beta voltage amplitudes, within the respective frequency ranges, at each recording electrode site. fTA and frontal beta asymmetries (fBA) were computed as the mean voltage recorded at electrode F7 minus the mean voltage recorded at electrode F8. The F7 and F8 electrodes are standard recording sites overlying the frontal lobes of the left and right cerebral hemispheres, respectively.

Statistical significance of differences in TBR, fTA and fBA between the two block examinations was determined using the 2013 Microsoft Excel statistical software package. Statistical significance was guaranteed by one-way analysis of variance (ANOVA) and post-hoc t-testing, at $\mathrm{P}<0.05$. Significance of correlations between TBR, fTA, fBA and the two examination scores was determined by computing the Pearson product-moment correlation coefficient and linear regression analysis, at $\mathrm{P}<0.05$.

\section{Results}

Figure 1 shows that fTA and fBA measured before the second examination were negatively correlated with TBR measured before the second examination $(\mathrm{r}=-0.74, \mathrm{P}<$ 0.03 and $\mathrm{r}=-0.60 \mathrm{P}<0.05$, respectively). Figure 2 shows that fTA and fBA were positively correlated with the second examination score $(\mathrm{r}=0.71, \mathrm{P}<0.03$ and $\mathrm{r}=0.54, \mathrm{P}=$ 0.1 , respectively). None of the qEEG measurements made before the first examination were found to be significantly correlated with the first examination score. 


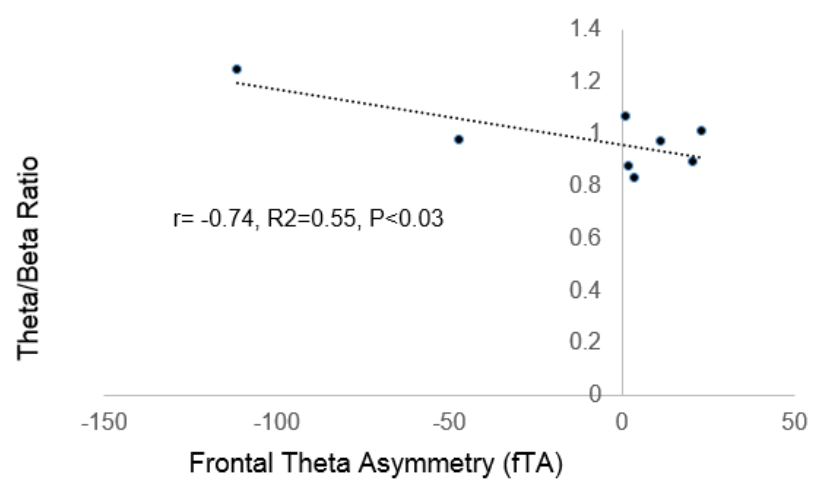

Figure 1 Correlation Between Frontal Theta Asymmetry and Theta-Beta Ratio. Trendline shows significant negative correlation between electroencephalographically-measured block 2 frontal theta asymmetry and block 2 theta-beta ratio. Subjects were nine male medical students studied $3 \mathrm{~d}$ before each of their first two Block Human Structure-Function (HSF) Course examinations covering integrated anatomy and physiology topics.

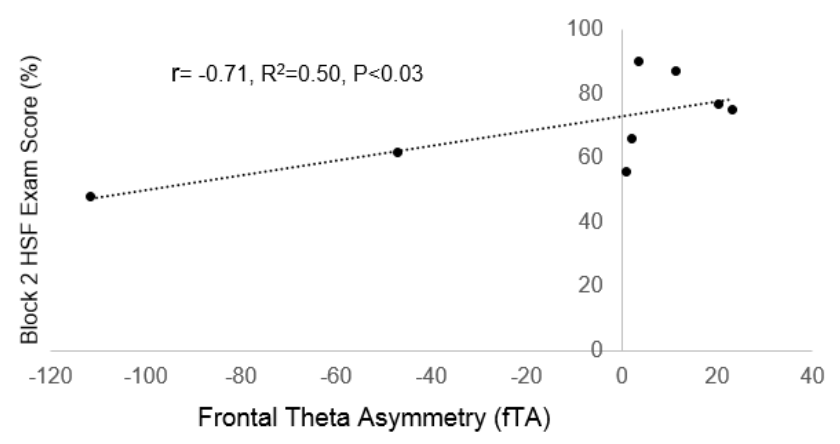

Figure 2 Correlation Between Frontal Theta Asymmetry and Examination Score. Trendline shows significant positive correlation between electroencephalographically-measured block 2 frontal theta asymmetry and block 2 examination score. Subjects were nine male medical students studied $3 \mathrm{~d}$ before each of their first two Block Human Structure-Function (HSF) Course examinations covering integrated anatomy and physiology topics.

\section{Discussion}

Our previous study demonstrated that TBR measured 3 $\mathrm{d}$ before the second of two first- semester block examinations covering integrated topics in anatomy-physiology, was negatively correlated with the second examination score [13]. The main finding of the present study using the same subjects and database, is that the second examination score was negatively correlated with the TBR measured 3 days prior to the exam and was positively correlated with frontal theta and beta asymmetries (fTA, fTB, respectively).

Since the 1970s, there has been increasingly more research linking EEG abnormalities with attention deficit disorders (ADD) [14]. There appear to be fairly consistent differences in the EEG patterns in most people with ADD compared to normal individuals. A meta-analysis demonstrates that an increase in the TBR power ratio is a reliable EEG finding in ADD [15]. Besides the potential for clinical ADD diagnosis, there is interest in the TBR in healthy adults as a measure of attention control [16].

The main drawback of conventional EEG is its inherent subjectivity, an impediment to using EEG as a research tool. The advent of qEEG technology has overcome this impediment by providing precise measurements of voltage within each of the standard EEG frequency bands of interest; delta, theta ( $\mathrm{T}$ ), alpha and beta (B). In conventional EEG, a trained observer, usually a physician, visually detects and reports abnormal waveforms. Such waveforms include spikes associated with epilepsy or uncharacteristic frequencies, usually slow waves, for identifying areas containing brain tissues injured by tumors, ischemia, infarct or stroke. qEEG differs from conventional EEG primarily by the data analysis. Because it is a time series, the analog data is decomposed to a fast Fourier transform (FFT), expressed in individual frequency bands, as a power spectrum. The FFT data for each electrode site on the scalp are expressed in voltage amplitude or power, as well as the dominant frequency for each of the four standard EEG frequency bands: delta $(2-4 \mathrm{~Hz})$, theta $(4-7 \mathrm{~Hz})$, alpha (8-12 $\mathrm{Hz})$ and beta $(13-30 \mathrm{~Hz})$. The qEEG is also shown to be useful for selecting those children and adults who are likely to be responsive to treatment with neurostimulant medications such as amphetamines [17-19]. qEEG is becoming more widely used for neurofeedback training in both athletics and academics. qEEG profiles indicative of improved athletic performance in major league baseball players and in Olympic athletes with performance deficits following injuries are reported [20]. qEEG-based neurofeedback training is also shown to be effective for improving neurosurgical skills in ophthalmic microsurgeons [21]. A relatively specific qEEG signature is shown to be indicative of working memory deficits in low-performing high school students, compared to their high- performing counterparts [22]. Specific qEEG changes, indicative of increased brain efficiency, are also shown to result from several practices of yoga [23, 24], suggesting a potential for yoga training to generate improvements in academic performance.

Evidence is emerging that TBR might provide an objective marker for prefrontally-mediated executive cognitive control (specifically, attentional control (AC) in healthy adults. There seems to be a negative relationship between TBR, expression of an AC trait and stress-induced AC decline, attentional orienting, emotional regulation, behavioral inhibition, and motivated decision-making in healthy adults [25]. These findings support the notion that stress or anxiety compromises cognitive executive performance. Previous studies have shown that TBR is related to trait attentional control, which could be an indicator of the effects of stress on attentional function [26]. The influence of stress on TBR and attentional control is reported to explain about one-fourth of the variance in stress-induced deterioration of self-reported attentional control [22]. In that regard, individuals with higher TBR are shown to exhibit larger declines of subjectively- experienced attentional control 
than those with lower TBR [26]. It is also reported that higher TBR is correlated with quick behavioral responses-to-choice stimuli, albeit with a high error rate [27].

The correlation between high TBR and poor attentional control or cognitive performance suggests the hypothesis that medical students with higher TBR exhibit poorer academic performance than those with lower TBR. Results of the present study support this hypothesis by demonstrating a significant negative correlation between TBR and the second of two first- semester anatomy-physiology course examinations. The correlation between TBR and examination score was found to be not significant for the first of the two examinations. We speculate that the EEG recording session before the first examination served to acclimate the subjects to the study protocol and that they were more proficient subjects during the session before the second examination. These qEEG sessions required the subjects to remain still with eyes closed for about ten minutes with a recording cap in place. In that regard, substantially more recording artifacts were observed in the first compared to the second recording session for all the subjects.

In humans, low theta wave oscillations dominate parts of the hippocampus during certain tasks. The amplitude of such low theta waves in the left temporal lobe is reported to increase in amplitude when viewing familiar objects or locations [27]. The left temporal area of the brain is also thought to be the seat of episodic memory [28]. The right hippocampus area shows increases in the amplitude of low theta oscillations during times of navigational demand [28]. Thus, both hippocampi appear to have various functions separate from each other and are involved in spatial and verbal activities.

These findings suggest that lateralized theta oscillations in left/right hippocampi play different roles in behavioral processes. A novel finding of the present study is that TBR measured before the second examination was positively correlated with both fTA and fBA and that fTA and ABA were positively correlated with the second examination score. fTA and fTB values were generally positive values, indicating larger theta and beta voltages in the left than in the right. This finding is consistent with that of the aforementioned study suggesting that left theta asymmetry is an indicator of memory functions and of viewing familiar objects and places, lateralized to the left hippocampus. In the present study, the subjects were seated at rest with eyes closed in front of the same blank wall $3 \mathrm{~d}$ before each of two block exams. The recording site could, therefore, be considered a familiar object and place for the second recording session $3 \mathrm{~d}$ before the students' second block examination. The latter qEEG measurements were significantly different from the former ones done $3 \mathrm{~d}$ before the first block examination.

It is reported that the human brain adapts optimally to intellectual challenges via a relaxed but alert, calm problem-solving mental state, with focus and concentration and without negative self- talk or circular thinking [28-30]. When a person achieves this mental state, there are corresponding changes in their EEGs, including lowering of their TBR ratios [28]. The results of the present study suggest that TBR, fTA and fBA in first-semester medical students may be indicators of academic performance. If these are reliable indicators, cognitive techniques should be able to use them to elucidate the mechanisms underlying adaptations to academic challenges, as well as those produced by pharmacopuncture treatments to improve cognitive deficits and academic performance.

\section{Conclusion}

These preliminary findings suggest that increases in theta and beta voltage amplitudes and decreases in the theta/ beta ratio (TBR) may characterize the qEEG signatures of first-semester male medical students between periods of preparation for their first two block examinations. These results also seem to support the hypothesis that high TBR and low frontal theta and beta asymmetries (fTA, fBA) are associated with low academic performance on block examination questions covering information presented in a first-semester anatomy-physiology course. These findings should be interpreted cautiously pending a larger study. In that regard, future studies with a larger number of subjects should determine whether these findings are gender- and/ or age- specific and if decreasing TBR and/or increasing fTA and fBA are predictors of higher performance in other academic challenges. Such studies should also determine the extent to which genetic profiles and specific genetic haplotypes are correlated with TBR, fTA, fBA and academic performance in medical school and other academic curricula. This experimental design may also serve as a model for identifying early cognitive deficits and for evaluating pharmacopuncture for improving cognitive functions in patients with neurological and neuropsychiatric disorders.

\section{Financial support and sponsorship}

This work is supported by a grant from the American University of Antigua College of Medicine Research Council

\section{Conflicts of interest}

The authors have no conflicts of interest to disclose 


\section{References}

1. Jittiwat J, Wattanathorn, Ginger J. pharmacopuncture improves cognitive impairment and oxidative stress following cerebral ischemia. J Acupunct Meridian Stud. 2012;5(6):295-300.

2. Isbell E, Stevens C, Pakulak E, Hampton Wray A, Bell TA, Neville HJ. Neuroplasticity of selective attention: Research foundations and preliminary evidence for a gene by intervention interaction. Proc Natl Acad Sci U S A. 2017;114(35):9247-54.

3. Dean AC, Morales AM, Hellemann G, London ED. Cognitive deficit in methamphetamine users relative to childhood academic performance: link to cortical thickness. Neuropsychopharmacology. 2018;43(8):1745-52.

4. Jolivet, Coggan JS, Allaman I, Magistretti PJ. Multi-timescale modeling of activity- Dependent metabolic coupling in the neuron-glia-vasculature ensemble. PLoS Computational Biology. 2015;11:e1004036.

5. Duyn JH. EEG-fMRI methods for the study of brain networks during sleep. Frontiers in Neurology. 2012;3:100. doi:10.3389/fneur.2012.00100.

6. Plichta M, Wolf I, Hohmann S, et al. Simultaneous EEG and fMRI reveals a causally connected subcortical-cortical network during reward anticipation. J Neurosci. 2013; 33(36):14526-33.

7. FDA News Release July 15, 2013.https://wayback.archiveit.org/7993/20170112223021/http://www.fda. gov/NewsEvents/ Newsroo m/PressAnnouncements/ ucm360811.htm.

8. Pavlov YG, Kotchoubey B. EEG correlates of working memory performance in females. BMC Neurosci. 2017;18(1):26. doi: 10.1186/s12868-017-0344-5.

9. Donner T H, Siegel M. A framework for local cortical oscillation patterns. Trends Cogn Sci. 2011;15(5):191-9.

10. Putman P, Verkuil B, Arias-Garcia E, Pantazi I, van Schie C. EEG theta/beta ratio as a potential biomarker for attentional control and resilience against deleterious effects of stress on attention. Cogn Affect Behav Neurosci. 2014;14(2):782-91.

11. Enriquez-Geppert S, Huster RJ, Figge C, Herrmann CS. Self-regulation of frontal-midline theta facilitates memory updating and mental set shifting. Front Behav Neurosci. 2014; 8:420. doi: 10.3389/fnbeh.2014.00420.

12. Kirov R, Weiss C, Siebner HR, Born J, Marshall L. Slow oscillation electrical brain stimulation during waking promotes EEG theta activity and memory encoding. Proc Natl Acad Sci U S A 2009;106(36):15460-5. doi: 10.1073/pnas.0904438106.

13. Gorantla VR, Parsons G, Sayed E, Fadel A, Olukoga C, Volkova YA, et al. Electroencephalographic correlates of brain adaptations to medical school academic challenges-a pilot study. J Clin Diagn Res. 2018;12(4):CC05-CC08.

14. Loo SK, Makeig S. Clinical Utility of EEG in Attention-Deficit/Hyperactivity Disorder: A Research Update. Neurotherapeutics. 2012;9(3):569-87.

15. Ogrim G, Kropotov J, Hestad K. The quantitative EEG theta/beta ratio in attention deficit/hyperactivity disorder and normal controls: Sensitivity, specificity, and behavioral correlates. Psychiatry Res. 2012;198(3):482-8. 16. Angelidis A, van der Does W, Schakel L, Putman P.
Frontal EEG theta/beta ratio as an electrophysiological marker for attentional control and its test-retest reliability. Biol Psychol. 2016;121(Pt A):49-52.

17. White JN, Hutchens TA, Lubar JF. Quantitative EEG assessment during neuropsychological task performance in adults with attention deficit hyperactivity disorder. J Adult Dev. 2005;12(2):113-21.

18. Sangal RB, Sangal JM. Use of EEG beta-1 power and theta/beta ratio over Broca's area to confirm diagnosis of attention deficit/hyperactivity disorder in children. Clin EEG Neurosci. 2015;46(3):177-82.

19. Snyder SM, Rugino TA, Hornig M, Stein MA. Integration of an EEG biomarker with a clinician's ADHD evaluation. Brain Behav. 2015;5:e00330.doi:10.1002/ brb3.330.

20. Graczyk M, Pąchalska M, Ziółkowski A, Mańko G, Łukaszewska B, Kochanowicz K, et.al. Neurofeedback training for peak performance. Ann Agri En vironl Med. 2014;21(4):871-5.

21. Ros T, Moseley MJ, Bloom PA, Benjamin L, Parkinson LA, Gruzelier JH. Optimizing microsurgical skills with EEG neurofeedback. BMC Neurosci. 2009;10:87. doi: 10.1186/1471-2202-10-87.

22. Aguirre-Pérez DM, Otero-Ojeda GA, Pliego-Rivero FB, Ferreira-Martínez AA. Relationship of working memory and EEG to academic performance: A study among high school students. Int J Neurosci. 2007;117(6):869-82.

23. Field T, Diego M, Hernandez-Reif M. Tai Chi/ Yoga Effects on Anxiety, Heartrate, EEG and Math Computations. Complement Ther Clinl Pract. 2010;16(4):235-38.

24. Trakroo M, Bhavanani AB, Pal GK, Udupa K, Krishnamurthy N. A comparative study of the effects of asan, pranayama and asan-pranayama training on neurological and neuromuscular functions of Pondicherry police trainees. Int J Yoga. 2013;6:96-103.

25. Putman P, van Peer J, Maimari I, van der Werff S. EEG theta/beta ratio in relation to fear- modulated response-inhibition, attentional control, and affective traits. Biol Psychol. 2010 83(2):73-8.

26. Putman P, Verkuil B, Arias-Garcia E, Pantazi I, Schie C. EEG theta/beta ratio as a potential biomarker for attentional control and resilience against deleterious effects of stress on attention. Cogn Affect Behav Neurosci. 2014;14(2), 782-91.

27. Miller J, Watrous AJ, Tsitsiklis M, Lee SA, Sheth SA, Schevon CA, et al. Lateralized hippocampal oscillations underlie distinct aspects of human spatial memory and navigation. Nat Commun. 2018;9(1):2423. doi: 10.1038/s41467-018-04847-9.

28. Hosseini S, Pritchard-Berman M, Sosa N, Ceja A, Kesler S. Task-based neurofeedback training: A novel approach toward training executive functions. Neuroimage. 2016;134:153-9.

29. Thompson M, Thompson L. Improving attention in adults and children: differing electroencephalography profiles and implications for training. Association for Applied Psychophysiology \& Biofeedback. 2006;34(3):99-105.

30. Vernon D, Egner T, Cooper N, Compton T, Neilands C, Sheri A, et al. The effect of training distinct neurofeedback protocols on aspects of cognitive performance. Int J Psychophysiol. 2003;47(1):75-85. 